\title{
DETERMINACIÓN DE ROJO DE PIROGALOL USANDO UN ELECTRODO DE CARBONO VÍTREO MODIFICADO CON PELÍCULA DE ORO Y CISTEAMINA POR VOLTAMPEROMETRÍA DE ADSORCIÓN (AdSV). POSIBLE USO COMO SENSOR ELECTROQUÍMICO
}

\begin{abstract}
Karen Bolaños*, Yaritza Leiva ${ }^{\mathrm{a}}$, Adrián Mariño ${ }^{\mathrm{a}}$, Claudia Cuéllar ${ }^{\mathrm{a}}$, Carolina Quimbaya ${ }^{\mathrm{a}}$, Paola Castiblanco ${ }^{\mathrm{a}}$, Manuel Otiniano ${ }^{\mathrm{b}}$, Edgar Nagles ${ }^{\mathrm{a}^{*} \text {, }}$
\end{abstract}

\section{RESUMEN}

Se estudió la interacción del Rojo de Pirogalol (RP) sobre la superficie de un electrodo de carbono vítreo modificado con película de oro (AuF) y cisteamina (cis). Teniendo presente el orden de la modificación, se encontró que la respuesta de oxidación del RP presentó mayor corriente con el electrodo modificado con cisteamina sobre película de oro electro-depositada sobre carbono vítreo $(\mathrm{GC} / \mathrm{AuF} / \mathrm{Cis} / \mathrm{E})$, el cual permite la oxidación de $\mathrm{RP}$ a un potencial de $0,48 \mathrm{~V}$ a pH 3,25. Con el método univariable se buscó parámetros óptimos, tales como $\mathrm{pH}$, velocidad de barrido $(\mathrm{U})$, potencial de adsorción $\left(\mathrm{E}_{\text {ads }}\right)$ y tiempo de adsorción $\left(\mathrm{t}_{\text {ads }}\right)$, obteniéndose valores de: $\mathrm{pH} 3,25 ; \mathrm{U} 75,50 \mathrm{mV} \mathrm{s}^{-1} ; \mathrm{E}_{\text {ads }} 0,10 \mathrm{~V} \mathrm{y} \mathrm{t}_{\mathrm{ads}} 60 \mathrm{~s}$, respectivamente. La curva de calibrado alcanzó un rango de linealidad entre $2,00 \times 10^{-7}$ y $15,14 \times 10^{-7} \mathrm{~mol} \mathrm{~L}^{-1}$. El límite de detección $(3 \mathrm{~S} / \mathrm{m})$ fue de 1,20 x 10-7 $\mathrm{molL}^{-1}$ y la desviación estándar relativa para siete mediciones iguales de RP 5,0 x $10^{-4} \mathrm{~mol} \mathrm{~L}^{-1}$ fue de $1,01 \%$.

Palabras clave: Rojo de pirogalol, voltamperometría de adsorción, cisteamina, película de oro.

\section{DETERMINATION OF PYROGALLOL RED ON A GLASSY CARBON ELECTRODE MODIFIED WITH GOLD FILM AND CYSTEAMINE BY ADSORPTION VOLTAMMETRY (AdsV). POSSIBLE USE AS ELECTROCHEMICAL SENSOR}

\begin{abstract}
Interaction pyrogallol red (RP) on glassy carbon electrode surface modified gold film (AUF) and cysteamine (cis) was studied. Bearing in mind the order of the modification, it was found that the response of oxidation of RP showed higher current to the electrode modified with gold film electro-deposited on cysteamine glassy carbon (GC/Cis / $\mathrm{AuF} / \mathrm{E})$, which allows the RP oxidation potential of $0.48 \mathrm{~V}$ at $\mathrm{pH} 3.25$. Electrochemical devices, such as $\mathrm{pH}$, scan rate $(\mathrm{U})$, adsorption potential $\left(\mathrm{E}_{\mathrm{ads}}\right)$ and adsorption time $\left(\mathrm{t}_{\mathrm{ads}}\right)$ parameters were optimized to yield values: $\mathrm{pH} 3.25 ; \mathrm{U} 75.50 \mathrm{mV} \mathrm{s}^{-1} ; \mathrm{E}_{\text {ads }} 0.10 \mathrm{~V}$ and $\mathrm{t}_{\text {ads }} 60 \mathrm{~s}$ respectively. The calibration curve linearity reached a range between $2.00 \times 10^{-7}$ and $15.14 \times 10^{-7} \mathrm{~mol} \mathrm{~L}^{-1}$. The detection limit $(3 \mathrm{~S} / \mathrm{m})$ was $1.20 \times 10^{-7} \mathrm{~mol} \mathrm{~L}^{-1}$ and relative standard deviation for measurements of seven equal RP $5.0 \times 10-4 \mathrm{~mol} \mathrm{~L}^{-1}$ was $1.01 \%$.
\end{abstract}

Key words: Red pyrogallol, adsorption voltammetry, cysteamine, gold film.

\footnotetext{
*a Universidad de la Amazonía, Facultad de Ciencias Básicas, Programa de Química, Florencia, Colombia. k.bolanos@udla.edu. co; ernagles@udla.edu.co

b Facultad de Química e Ingeniería, Universidad Nacional Mayor de San Marcos, Lima, Perú
} 


\section{INTRODUCCIÓN}

El rojo de pirogalol (figura 1) es un compuesto que se puede encontrar en la naturaleza, moléculas sintéticas y productos farmacéuticos. Estudios han revelado su gran capacidad y propiedades antioxidantes ${ }^{1}$. Por otro lado, es utilizado para la cuantificación de proteínas tales como la de Bence Jones ${ }^{2}$ y proteinuri ${ }^{3}$, así como también en pruebas de actividad antioxidante debido a su alta reactividad en presencia de radicales libres ${ }^{4}$. Recientemente, se ha usado como complejante de metales; es el caso del indio $^{5}$, aluminio ${ }^{6}$, cadmio, plomo $^{7}$, antimonio ${ }^{8}$, cobre y molibdeno 9 . Presentando límites de detección igual y menor de $1 \mu \mathrm{g} \mathrm{L}^{-1}$.

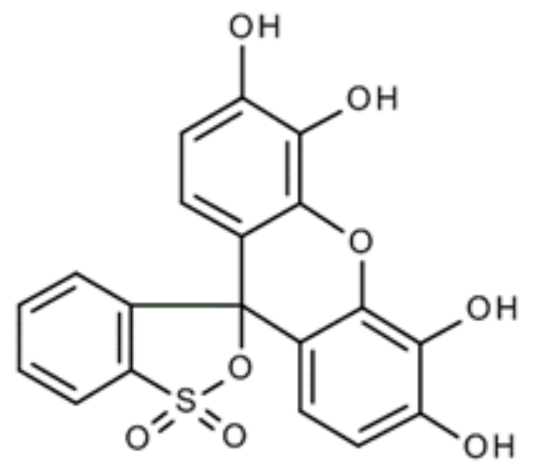

Figura 1. Estructura química del rojo de pirogalol.

Tratándose de una sustancia con capacidad antioxidante ${ }^{1}$ y debido a la aplicación y el enfoque cuantitativo que se le ha dado a este compuesto, es necesaria la implementación de técnicas sensibles para su cuantificación: en este caso se hará uso de técnicas electroquímicas y desarrollo de electrodos modificados como herramienta fundamental en la detección del RP.

AdSV es una técnica electroanalítica muy sensible, que involucra un paso de preconcentración del analito sobre la superficie del electrodo y un segundo paso de barrido del analito depositado usando voltametría de onda cuadrada (SWV). Dos componentes necesarios en este estudio afectan la respuesta voltamperométrica: la solución electrolítica y la superficie del electrodo; por lo tanto, parte del estudio consiste en la optimización del pH y la caracterización de electrodos modificados (con voltametría cíclica) con el fin de mejorar la señal del RP.

Estudios anteriores han reportado un aumento en la sensibilidad del electrodo atribuida a la formación de una monocapa, gracias a la alta afinidad entre el azufre del grupo tiol de la cisteamina y el oro ${ }^{10}$; por lo tanto las modificaciones fueron realizadas con $\mathrm{HAuCl}_{4}$ y cisteamina. Este tipo de modificaciones se emplea principalmente en el desarrollo de biosensores, porque ofrece la posibilidad de incorporar grupos funcionales que den lugar a la interacción química con las enzimas ${ }^{11}$ y en el aumento de la sensibilidad en la cuantificación de iones metálicos de soluciones acuosas puesto que aumenta la fijación de los mismos ${ }^{12}$. Se ha reportado el uso de electrodos modificados con cisteamina y oro para la determinación de ácido úrico ${ }^{13}$, pero en se ha reportado aún para rojo de pirogalol. 


\section{Reactivos}

\section{PARTE EXPERIMENTAL}

El agua usada para lavar y preparar disoluciones fue agua desionizada $18 \mathrm{M} \Omega \mathrm{cm}$ de un sistema milliQ. Las disoluciones patrones con $2,50 \times 10^{-3}$ y $2,27 \times 10^{-4} \mathrm{molL}^{-1}$ de rojo de pirogalol fueron preparadas a partir del reactivo puro de Aldrich-Sigma y fue preparado en agua. Las disoluciones de buffers $0,10 \mathrm{M}$ fosfato $\left(\mathrm{H}_{2} \mathrm{PO}_{4}^{-} / \mathrm{H}_{3} \mathrm{PO}_{4}\right)$ y acetato $\left(\mathrm{CH}_{3} \mathrm{COO}^{-} /\right.$ $\mathrm{CH}_{3} \mathrm{COOH}$ ) fueron preparadas a partir de sus ácidos y sales respectivas y ajustando el $\mathrm{pH}$ requerido con disolución de $\mathrm{NaOH} 2,0 \mathrm{~mol} \mathrm{~L}^{-1}$ y diluyendo 10 veces con agua.

\section{Instrumentos y equipos}

Para el desarrollo de las medidas voltamperométricas se utilizó un analizador voltamétrico (VA Computrace 997 Metrohm), para la adquisición de datos. La celda de $10 \mathrm{~mL}$ consta de un sistema de tres electrodos: un electrodo de carbono vítreo de $3 \mathrm{~mm}$ de diámetro como electrodo de trabajo, un electrodo de referencia de $\mathrm{Ag} / \mathrm{AgCl} / \mathrm{KCl} 3 \mathrm{~mol} \mathrm{~L}^{-1}$ y un alambre de platino como electrodo auxiliar. En la preparación de los buffers se utilizó un peachímetro Orion 430 con electrodo de membrana de vidrio.

\section{Procedimiento}

\section{Modificación del electrodo}

La figura 2 ilustra la modificación del electrodo de trabajo (carbono vítreo). La adsorción de cisteamina, el electrodo se sumergió en una solución de cisteamina $10 \mu \mathrm{g} \mathrm{L}{ }^{-1}$ durante 1 hora a $60^{\circ} \mathrm{C}$. Después de este tiempo, se lavó el electrodo con agua miliQ para eliminar el exceso de solvente. Luego el electrodo modificado con cisteamina de colocó en una celda que contenía $\left(\mathrm{HAuCl}_{4}\right) \cdot 2 \mathrm{H}_{2} \mathrm{O}$ de $100 \mathrm{mg} \mathrm{L}{ }^{-1}$. La electrodeposición de oro se realizó con agitación constante a $500 \mathrm{rpm}$ con un tiempo y potencial de depósito de $150 \mathrm{~s}$ y $-0,20 \mathrm{~V}$, respectivamente.

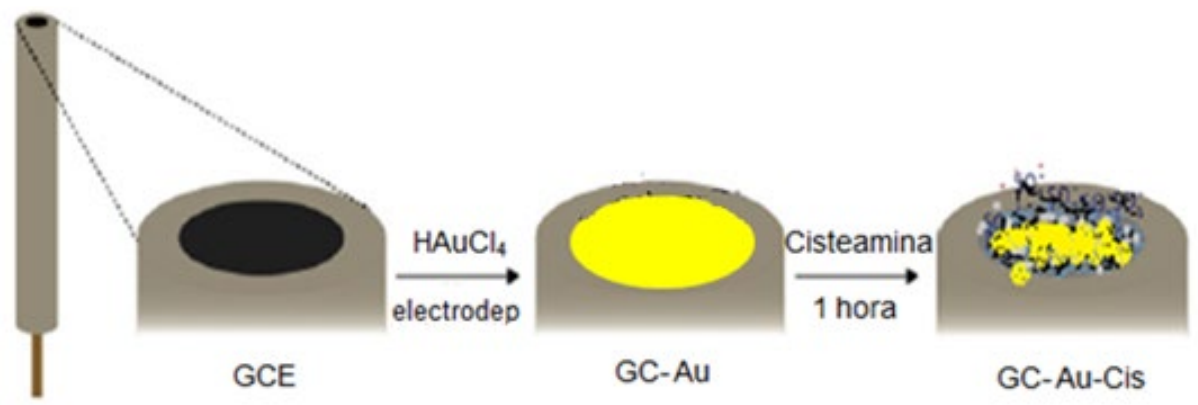

Figura 2. Modificación del electrodo de trabajo.

\section{Optimización de parámetros por método univariable}

En busca de parámetros óptimos se estudio la corriente de pico en función del $\mathrm{pH}$ entre 2,20 y 3,49 con el sistemas buffer fosfato, el potencial de adsorción (Eads) entre 0,1 y 1 V bajo las mismas condiciones. Teniendo el potencial óptimo de adsorción se varió el tiempo de adsorción (tads) de 10 - $100 \mathrm{~s}$ buscando un incremento en la corriente de pico con el método univariable. Se calculó el límite de detección $(\mathrm{LOD}=3 \sigma)$ y se midió siete muestras idénticas para determinar la desviación estándar relativa. 


\section{Caracterización de electrodos modificados}

\section{RESULTADOS Y DISCUSIÓN}

La figura 3 muestra los voltamperogramas cíclicos de RP sobre diferentes electrodos modificados de GC. Electrodo sin modificar (GC/E, curva 1); modificado con oro y cisteamina (GC/AuF/Cis/E, curva 2) y modificado con cisteamina y oro (GC/Cis/AuF/E, curva 3). A 100 $\mathrm{mV} \mathrm{s}^{-1}$ en buffer fosfato $\mathrm{pH} \mathrm{3,25.}$

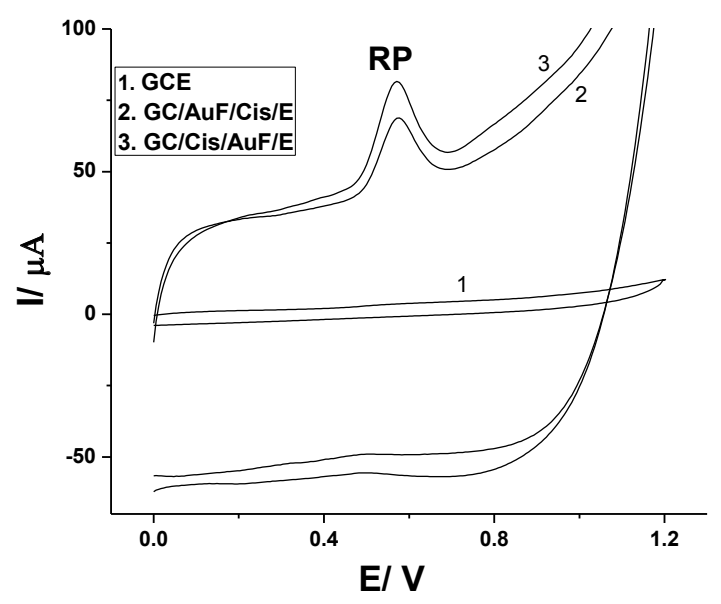

Figura 3. Voltamperogramas cíclicos del RP $0,30 \times 10^{-3} \mathrm{~mol} \mathrm{~L}^{-1}$. Electrodo sin modificar (GC/E, curva 1$)$; modificado con oro y cisteamina $(\mathrm{GC} / \mathrm{AuF} / \mathrm{Cis} / \mathrm{E}$, curva 2$)$ y modificado con cisteamina y oro (GC/Cis/AuF/E, curva 3). $\mathrm{pH} \mathrm{3,25} \mathrm{y} \mathrm{velocidad} \mathrm{de} \mathrm{barrido} \mathrm{de} 0,1 \mathrm{mVs}^{-1}$.

La corriente de pico de la oxidación de RP fue más alta con el electrodo GC/Cis/AuF/E. El posible aumento de la corriente con el electrodo GC/Cis/AuF (curva 3) puede explicarse por la gran afinidad entre el azufre de la cisteamina y la película de oro, permitiendo una mejor superficie de la película de oro depositada y, de esta manera, la cantidad de RP depositada fuera mayor comparada cuando se deposita sobre cisteamina. El electrodo modificado GC/ $\mathrm{Cis} / \mathrm{AuF} / \mathrm{E}$ se usó para estudios posteriores.

\section{Estudio en función del pH por voltamperometría de adsorción (AdSV)}

En la figura 4 se evidencia la variación de la corriente de pico en función del pH de una disolución de RP 0,3x10-5 mol L-1. Se observa que el pH óptimo es 3,25 ( $\mathrm{H}_{3} \mathrm{PO}_{4} / \mathrm{H}_{2} \mathrm{PO}_{4}{ }^{-}$Esto se puede explicar en función de los valores de $\mathrm{pKa}$ del RP. El RP tiene $\mathrm{pKa} \mathrm{a}_{1}: 6,2 ; \mathrm{pKa}_{2}: 8,9$ y $\mathrm{p} K \mathrm{a}_{3}: 11,9^{14}$ y cuanto más cercano sea el valor de $\mathrm{pH}$ de la disolución a los valores de $\mathrm{p} K \mathrm{a}_{1,2,3}$ del RP, hay mayor posibilidad de desprotonar los grupos -OH del RP. A valores mayores de $\mathrm{pH}$ la corriente desciende indicando la desprotonación de los $\mathrm{OH}^{-}$del $\mathrm{RP}^{6}$. El pH 3,25 se selecciona como óptimo y se usó en los estudios posteriores. 


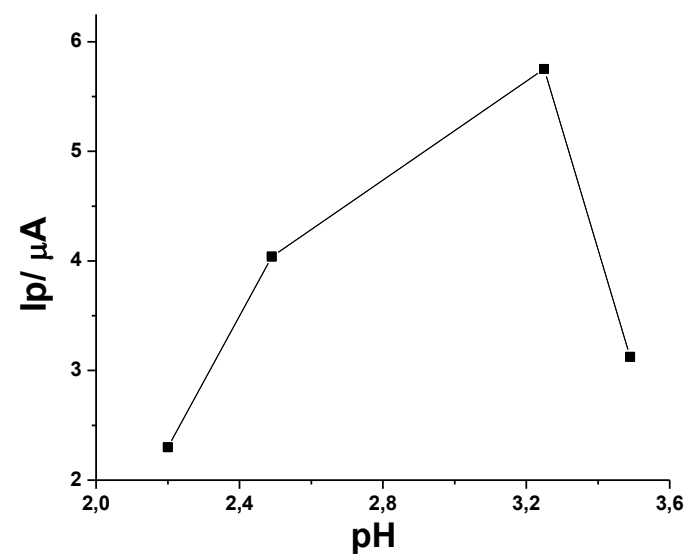

Figura 4. Estudio del RP en función del pH. Sobre GC/Cis/AuF/E. RP 0,3 x 10-5 $\mathrm{mol} \mathrm{L}^{-1}$. Condiciones: tacc 60s; Eacc 0.1V; Frecuencia $20 \mathrm{~Hz}$.

Estudio en función del tiempo de acumulación ( $\left.t_{\text {acm }}\right)$ y potencial de acumulación $\left(E_{a c m}\right)$. El tiempo y potencial de acumulación son parámetros igual de importantes comparados con el pH. Estos están relacionados con el aumento de la sensibilidad permitiendo el depósito por adsorción del RP en la superficie del electrodo. A potenciales muy positivos o negativos en RP se deposita por procesos electrolíticos y no por adsorción; a tiempos muy altos se satura la superficie del electrodo afectando la adsorción de RP. Estudios previos con RP $0,3 \times 10^{-5}$ mol L-1 sobre el electrodo modificado $\mathrm{GC} / \mathrm{Cis} / \mathrm{AuF} / \mathrm{E}$, permite considerar un tiempo de $60 \mathrm{~s} \mathrm{y}$ un potencial de $0,1 \mathrm{~V}$ como tiempo y potencial de acumulación óptimos. Esto se realizó a pH 3.25. Estudios donde el RP se ha usado como ligando de metales han reportado un tiempo de acumulación entre 60 y $90 \mathrm{~s}$ como óptimo ${ }^{6-9}$

\section{Efecto de la velocidad de barrido (U)}

Se estudio el efecto de la velocidad de barrido y se graficó la raíz cuadrada de la velocidad de barrido $(\mathrm{U})^{1 / 2}$ vs la corriente de pico de $2,0 \times 10^{-4} \mathrm{molL}^{-1}$ de RP entre 5 y $100 \mathrm{mV} \mathrm{s}^{-1}$. Los resultados muestran un incremento lineal de la corriente de pico entre 10 y 70 $\mathrm{mV}(\mathrm{U})^{1 / 2}\left(\mathrm{R}^{2}=98,8\right)$; indicando que el proceso electroquímico es controlado por adsorción. A valores mayores de $(\mathrm{U})^{1 / 2}$, la corriente de pico decrece, indicando que la transferencia de carga es lenta. La variación del cociente $\mathrm{Ip} / \mathrm{C}$ donde Ip es la corriente de pico y $\mathrm{C}$ la concentración, no se mantiene constante para procesos controlados por adsorción. En este caso presenta una relación no lineal entre Ip/C, ya que al disminuir la concentración aumenta el cociente Ip/ $\mathrm{C}^{15}$. De acuerdo con la relación entre la corriente de pico $\left(\mathrm{i}_{\mathrm{p}}\right)$ y la carga $(\mathrm{Q})$ por un sistema de adsorción irreversible :

$$
\mathrm{ip}_{\mathrm{p}}=\mathrm{n}^{2} \mathrm{~F}^{2} \mathrm{~A} \Gamma \mathrm{U} / 4 \mathrm{RT}=\mathrm{nQF} \mathrm{U} / 4 \mathrm{RT}
$$

(Donde $\mathrm{A}$ es el área del electrodo, $\Gamma$ es la cantidad de adsorción de la superficie, $\mathrm{F}$ la constante de Faraday y $\mathrm{U}$ es la velocidad ${ }^{16}$. 
Manteniendo las condiciones constantes, Q (carga) es igual al área de la corriente de pico. El número de electrones transferidos calculado es el 1,86, indicando que se transfieren 2 electrones. Este dato es similar al reportado en trabajos previos ${ }^{17}$

\section{Parámetros de la técnica en la etapa de barrido}

Al igual que en los estudios anteriores se eligió una frecuencia de $20 \mathrm{~Hz}$, un potencial del escalón de $5 \mathrm{mV}$ y una amplitud del pulso de $40 \mathrm{mV}$. A otros valores diferentes se pierde la forma del pico de oxidación.

\section{Construcción de curva de calibrado}

La figura 5 A muestra los voltamperogramas de adsorción en función de la concentración de RP; la figura B es la curva de calibrado. Éstas fueron obtenidas con los parámetros óptimos (tabla 1). La curva de calibrado de RP tiene un rango de linealidad entre 2,00 x $10^{-7}$ y $15,14 \times 10^{-7} \mathrm{~mol} \mathrm{~L}^{-1}$.

Tabla 1. Parámetros experimentales aplicados en la obtención de los voltamperogramas y curva de calibrado del RP.

\begin{tabular}{lcc}
\hline Técnica & $\begin{array}{c}\text { AdSV } \\
\text { Electrodo }\end{array}$ & 3 \\
Diámetro $\left(\mathrm{mm}^{2}\right)$ & 550 \\
Velocidad de agitación(rpm) & 0,10 \\
Potencial de acumulación & $(\mathrm{V})$ & 60 \\
Tiempo de acumulación & $(\mathrm{s})$ & 10 \\
Tiempo de equilibrio & $(\mathrm{s})$ & 0,0 \\
Potencial de inicial & $(\mathrm{V})$ & 1,0 \\
Potencial final & $(\mathrm{V})$ & 5 \\
Potencial de escalón & $(\mathrm{mV})$ & 40 \\
Amplitud de pulso & $(\mathrm{mV})$ & 20 \\
Frecuencia & $(\mathrm{Hz})$ & \\
\hline
\end{tabular}

El límite de detección según el método Milller and Miller ${ }^{18}$ fue de 1,20 x $10^{-7}$ y la desviación estándar relativa para siete mediciones iguales de RP 5,0 x 10-4 $\mathrm{mol} \mathrm{L}^{-1}$ fue de $1,01 \%$ 

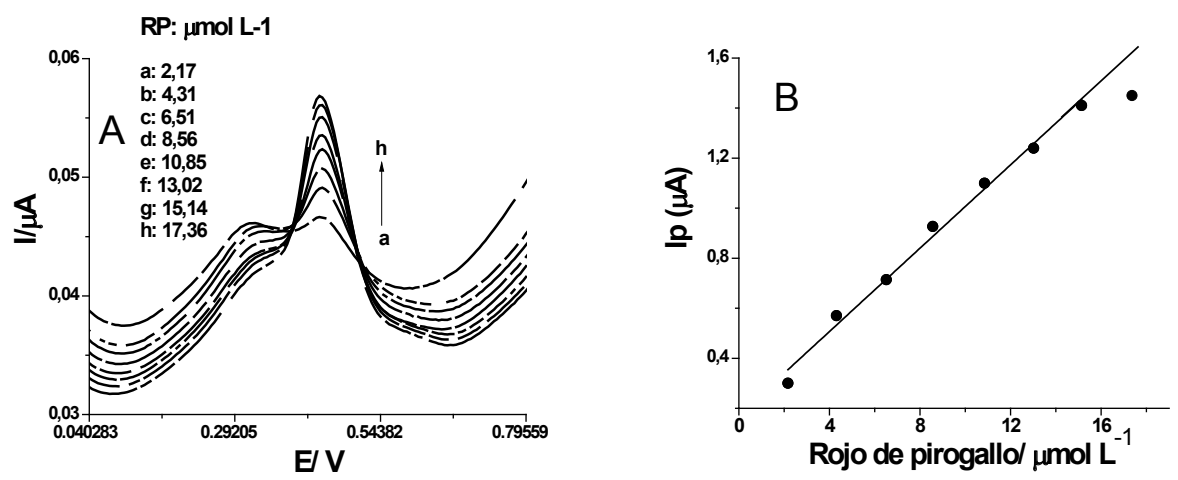

Figura 5. Voltamperogramas de adsorción en un rango de concentración 2 - $18 \mu \mathrm{molL}^{-1}$ A) y curva de calibrado B) de RP; pH: 3,2; $\mathrm{t}_{\text {ads }}: 60 \mathrm{~s} ; \mathrm{E}_{\text {ads }} 0,10 \mathrm{~V}$.

\section{CONCLUSIONES}

Se modificó electrodos y se caracterizaron mediante la técnica de AdsV y CV. Se determinó que el mejor electrodo para la determinación de RP fue el electrodo de cisteamina sobre oro, debido a la alta afinidad entre el analito y los componentes del electrodo. El método propuesto es sencillo, de bajo costo y puede ser usado en el desarrollo de sensores usados para determinar rojo de pirogalol en diversas matrices, como alimentos o productos naturales.

\section{AGRADECIMIENTOS}

Los autores agradecen a la Universidad de la Amazonía y a la Vicerrectoría de Investigación por el financiamiento del Proyecto de semillero de investigación 2014.

\section{BIBLIOGRAFÍA}

1. E. Atala, A. Aspeé, H. Speisky, E. Lissi \& C. López-Alarcón. Antioxidant capacity of phenolic compounds in acidic medium: A pyrogallol red-based ORAC (oxygen radical absorbance capacity) assay. J. Food Compos. Anal. 2013; 32: 116-125.

2. L. Madalena, M. Facio, A. Dragovetsky, D. Bresciani \& S. Fraind. Utilidad del método de rojo de pirogalol molibdato en la cuantificación de proteína de Bence Jones. Acta bioquímica clínica latinoamericana. 2002; 36 (1): 57-65.

3. L. Madalena, M. Facio, P. Bresciani, M. Pandolfo, C. Garlatti, M. Alejandre, M. Angerosa \& M. Pizzolato. Proteinuria: lesión estructural renal y comparación de métodos. Acta Bioquímica Clínica Latinoamérica. 2013; 47 (1): 85-93.

4. Pérez F. Estudio de la capacidad antioxidante de derivados de hidroxicumarina en sistemas homogéneos y en modelos de membrana lipídica. (Tesis doctoral). Programa: doctorado en química. Universidad de Chile, 2014

5. J. C. Aguilar, E. Rodríguez \& J. de Gyves. Adsorptive stripping voltammetry of In(III) in the presence of pyrogallol red in chloride-acetate media. Rev. Soc. Quím. Méx. 2001; 45 (1): 17.

6. V. Arancibia \& C. Muñoz C. Determination of aluminium in water samples by 
adsorptive cathodic stripping voltammetry in the presence of pyrogallol red and a quaternary ammonium salt. Talanta. 2007; 73(3): 546-552.

7. E. Nagles, R. Ríos, J. Hurtado \& V. Arancibia. Estudio electroquímico de Rojo de Pirogalol (RP) en presencia de surfactantes de diferente carga. Ingenium.2012; 6 (13): 65-68.

8. T. Kubota, A. Kawakami, T. Sagara, N. Ookubo \& T. Okutani. Determination of antimony content in natural water by graphite furnace atomic absorption spectrometry after collection as antimony (III)-pyrogallol complex on activated carbon. Talanta. 2001;53: 1117-1126.

9. A. Ensafi, T. Khayamian \& S. Shokooh. Application of adsorptive cathodic differential pulse stripping method for simultaneous determination of copper and molybdenum using pyrogallol red. Anal. Chim. Acta. 2004; 505(2): 201-207.

10. E. Herrera, M. Stragliotto \& C. Giacomelli C. caracterización de mono capas auto ensambladas de cisteamina sobre oro gold. En: Jornada; V Jornadas de Posgrado en Escuela de Posgrado de la Facultad de Ciencias Químicas, UNC. Córdoba, 2011.

11. L. Agüí, M. L. Mena, A. Gonzales \& P. Sedeño. Biosensores electroquímicos basados en el empleo de nanopartículas de oro. Anales de la real sociedad española de química julio-septiembre 2005 12-16.

12. I. Rendón \& J. Aguilar. Estudio Electroquímico de Mono capas Auto ensambladas de Cisteamina funcionalizadas con 6-metil-2-piridincarboxaldehído. Química Central. 2011; 2 (1): 33-38.

13. A.S. Ramírez-Segovia, J.A. Banda-Alemán, S. Gutiérrez-Granados, A. Rodríguez, F.J. Rodríguez, Luis A. Godínez, E. Bustos, J. Manríquez. Glassy carbon electrodes sequentially modified by cysteamine-capped gold nanoparticles and poly (amidoamine) dendrimers generation 4.5 for detecting uric acid in human serum without ascorbic acid interference. Anal. Chim. Acta (2014);812: 18-25

14. M. V. Ivanov \& A. M. Mamedov. 3, 4, 5-Trihydroxyfluorones as analytical reagents. J. Anal. Chem., 2006; 61(11): 1040-1062

15. S. Bedioui, A. Gutiérrez, J. Alatorre, J. Zagal. "Electroquímica. Voltametría sobre electrodos sólidos", primera edición, editorial USACH. Santiago, Chile, 2009, 145.

16. X. Ping, Q. Zhou, F. Xiao, F. Zhao \& B. Zeng. Sensitive Voltammetric Determination of Morin on a MultiWalled Carbon Nanotubes-Paraffin Oil Paste Electrode. Int. J. Electrochem. Sci., 2006; 1:28-237.

17. E. Atala, G. Velásquez, C. Vergara, C. Mardones, J. Reyes, R. A. Tapia, F. Quina, M. A. Mendes, H. Speisky, E. Lissi, M. S. Ureta-Zañartu, A. Aspée, \& C. López-Alarcón. Mechanism of Pyrogallol Red Oxidation Induced by Free Radicals and Reactive Oxidant Species. A Kinetic and Spectroelectrochemistry Study. J. Phys. Chem. B, 2013; 117 (17): 4870-4879

18. Miller J.C \& Miller J.N. Estadística y Quimiometría para Química Analítica. ADDISON-WESLEY. 2002; Cuarta edición: 124-125. 